\title{
A novel method for the nitration of deactivated aromatic compounds $\uparrow$
}

\author{
Keith Smith, ${ }^{* a}$ Tracy Gibbins, ${ }^{a}$ Ross W. Millar ${ }^{b}$ and Robert P. Claridge ${ }^{b}$ \\ ${ }^{a}$ Centre for Clean Chemistry, University of Wales Swansea, \$ Swansea, UK SA2 8PP \\ ${ }^{b}$ Defence Evaluation Research Agency, Fort Halstead, Sevenoaks, Kent, UK TN14 7 BP
}

\author{
Received (in Cambridge, UK) 16th March 2000, Accepted 13th June 2000 \\ Published on the Web 19th July 2000
}

\begin{abstract}
Novel nitration systems comprising nitric acid, trifluoroacetic anhydride and zeolite $\mathrm{H} \beta$, with or without acetic anhydride, are described. The system having no acetic anhydride is more active and readily nitrates deactivated substrates such as nitrobenzene, benzonitrile, benzoic acid or 4-nitrotoluene to give predominantly the product substituted meta to the deactivating group.

The system incorporating acetic anhydride is more moderate in activity, and nitrates moderately deactivated systems such as halogenobenzenes quantitatively within two hours at ice-salt bath temperature, and provides very high selectivity for the para-nitrated product. This system also nitrates 2-nitrotoluene to give high selectivity for 2,4-dinitrotoluene production. Furthermore, the system can be used for direct double nitration of toluene and gives a $92 \%$ yield of 2,4-dinitrotoluene with a 2,4-:2,6-dinitrotoluene ratio of $25: 1$. Even greater selectivity (96\% yield and 70:1 selectivity) can be achieved in the latter reaction by conducting the reaction in one flask but in two stages, with trifluoroacetic anhydride added only in the second stage.
\end{abstract}

\section{Introduction}

The industrially important process of nitration is probably the most studied of all the electrophilic aromatic substitution reactions ${ }^{1}$ and yet industry still relies on early technology utilising large amounts of strong mineral acids, which has many disadvantages. Nitration reactions are notoriously unselective; they often lead to by-product formation from oxidation of the substrate; the acids are highly corrosive and used in excess; and work-up requires an aqueous washing step resulting in the production of acidic effluent which is environmentally unfriendly and costly to treat. $^{2}$ However, with increasing pressure on industry to minimise the environmental impact of its processes, research has intensified on the replacement of the bulk acidic media by an acidic solid. As well as potentially catalysing a reaction, allowing it to proceed under milder, safer conditions, zeolites offer the possibility of influencing the regioselectivity of the reaction and minimising the environmental impact of the process.

Acidic solids have been used for selective nitration reactions, in particular the mononitration of aromatic compounds. For example, claycop (copper(II) nitrate supported on K10 montmorillonite clay) has been used to nitrate toluene with high para selectivity in the presence of acetic anhydride in tetrachloromethane. ${ }^{3}$ More recently, the highly acidic zeolite $\mathrm{H} \beta$ has been found to catalyse the nitration of simple aromatic substrates, for example toluene, by a mixture of nitric acid and acetic anhydride at room temperature and provides improved selectivity towards the para position over traditional mixed-acid systems. ${ }^{4}$ This system offers excellent possibilities for nitration of substrates which are moderate in activity. However, it is not very successful with deactivated substrates.

We now report further research in this field, with the focus on the nitration of deactivated substrates and on the dinitration of toluene with regioselectivity towards 2,4-dinitrotoluene. Indeed, we have been able to develop an optimised nitration

$\uparrow$ C Crown Copyright 2000

t Formerly known as the University College of Swansea. system for the nitration of such deactivated aromatic substrates and for highly selective dinitration of toluene to give 2,4dinitrotoluene.

Normally, the nitration of deactivated compounds (and therefore the polynitration of toluene) is carried out using aggressive nitric acid-oleum mixtures. Nitroaromatics such as 1,3-dinitrobenzene and 2,4-dinitrotoluene are important intermediates for the production of industrially important chemicals and are used in the preparation of polymers, explosives, dyes, pharmaceuticals, agrochemicals and fragrances. ${ }^{5}$

The dinitration of toluene with mixed acids produces 2,4- and 2,6-dinitrotoluenes in a ratio of $4: 1$, from which 2,4dinitrotoluene is isolated for the manufacture of toluene diisocyanate (TDI) and toluenediamine, both of which are used in the manufacture of polyurethanes. Zirconium and hafnium derivatives have been reported to be useful catalysts for the nitration of $o$-nitrotoluene, but ratios of 2,4-:2,6-dinitrotoluene were modest $(66: 34) .{ }^{6}$ Nitrogen dioxide has been used to polynitrate aromatic compounds at low temperatures in the presence of ozone and a catalyst such as methanesulfonic acid. $^{7}$ For example, nitrobenzene was nitrated to give a $99 \%$ yield of dinitrobenzene with regioselectivity $o: m: p 8: 91: 1 .^{7}$

A patent has reported the use of metal nitrates supported on clays for the nitration of aromatic compounds. ${ }^{5}$ One of the examples quoted was the dinitration of toluene using claycop, acetic anhydride, and nitric acid in the presence of carbon tetrachloride, producing dinitrotoluenes in a yield of $85 \%$ with a ratio of 2,4-:2,6-dinitrotoluene of $9: 1 .^{5}$ The degree of nitration achieved depended on the amount of modified clay used and the reaction time. This method, however, requires a large excess of nitric acid, the use of carbon tetrachloride and long reaction times ( 6 hours in the case of the dinitration of toluene). Other examples quoted included the production of 1,3-dinitrobenzene from benzene in a 70\% yield after 12 hours at room temperature. ${ }^{5}$

The highest recorded selectivity for the direct nitration of toluene to 2,4-dinitrotoluene involves nitric acid over a zeolite $\beta$ catalyst and gives a $2,4: 2,6$ ratio of $14{ }^{8}$ Full results are yet to be published. 
After our success in nitrating moderately active monosubstituted benzenes with benzoyl nitrate ${ }^{9}$ and acetyl nitrate, ${ }^{4}$ which are not reactive enough to take the nitration of toluene further than the first step, we decided to try trifluoroacetyl nitrate, which can be generated in situ from trifluoroacetic anhydride and nitric acid [eqn. (1)].

$$
\left(\mathrm{CF}_{3} \mathrm{CO}\right)_{2} \mathrm{O}+\mathrm{HNO}_{3} \rightleftharpoons \mathrm{CF}_{3} \mathrm{C}(\mathrm{O}) \mathrm{ONO}_{2}+\mathrm{CF}_{3} \mathrm{CO}_{2} \mathrm{H}
$$

Although trifluoroacetyl nitrate is known to be more active than acetyl nitrate, it has not been widely used in nitration reactions. ${ }^{1}$ However, unreactive aromatic substrates, such as nitrobenzene and bromobenzene, have been successfully nitrated using fuming nitric acid and trifluoroacetic anhydride in equimolar proportions at $45-55^{\circ} \mathrm{C} .{ }^{10} \mathrm{~m}$-Dinitrobenzene was formed in $65 \%$ yield from nitrobenzene, and bromobenzene was nitrated to give a mixture of nitrated products ( $o$-nitro(1\%), p-nitro- $(24 \%)$, and the 2,4-dinitro-derivative $(60 \%)){ }^{10}$ More reactive aromatic compounds such as anisole yielded predominantly diazonium salts when treated in a similar way. ${ }^{10}$ Toluene gave only traces of diazonium salts and no dinitration was reported..$^{10}$

Our hope was that the use of a zeolite might enhance the yields and/or selectivities available in nitrations of deactivated substrates with trifluoroacetyl nitrate generated according to eqn. (1). We now report that this is indeed the case.

\section{Results and discussion}

\section{Preliminary investigations}

Our initial studies involved the nitration of $p$-nitrotoluene, which nitrates exclusively to give 2,4-dinitrotoluene. We found that the trifluoroacetyl nitrate mixture [eqn. (1)] was active enough to nitrate this substrate in high yield at room temperature without the need for a zeolite. In the case of the nitration of $o$-nitrotoluene, there were two main products, 2,4-dinitrotoluene and 2,6-dinitrotoluene. Again, we found that the trifluoroacetyl nitrate mixture alone was active enough to effect nitration. However, the presence of $\mathrm{H} \beta$ did improve the regioselectivity slightly towards the 2,4-isomer $(2,4: 2,6$ ratio $3: 1$ compared to $2: 1$ without the zeolite), although it did not affect the overall reaction rate or the product yield very much.

There are two possible explanations for the lack of catalysis by the zeolite. 1) The pores of the zeolite are too small for the substrate to enter or for the dinitrated products to be formed within them. 2) The free solution reaction occurs so fast that the bulk of the reactants do not have time to diffuse into the pore system and so reaction occurs primarily in free solution or at the external surface of the zeolite.

If the latter explanation were correct, it should be possible to slow down the overall reaction by addition of a diluent such as dichloromethane or acetic anhydride, thereby giving the zeolite a better chance to exert an influence over the reaction. Indeed, addition of acetic anhydride led to a slower reaction and zeolite $\mathrm{H} \beta$ then exerted a greater influence over both the rate and selectivity.

The reaction was performed in the presence of a series of different zeolites under identical conditions and $\mathrm{H} \beta$ was found to be the best zeolite for regioselective nitration. Also, only a stoichiometric amount of nitric acid and the minimum amount of trifluoroacetic anhydride to achieve quantitative conversion to trifluoroacetyl nitrate were needed for complete reaction. This system was therefore chosen for more detailed study.

\section{Nitration of $o$-nitrotoluene}

Of the two main products from the nitration of $o$-nitrotoluene the 2,4 -isomer is the more commercially desirable. Classically, using mixed acids, the ratio of 2,4-:2,6-isomers is about $2: 1$.
Table 1 Effect of amount of acetic anhydride ${ }^{a}$

\begin{tabular}{lllll}
\hline & & \multicolumn{3}{l}{ Proportions of isomers } \\
\cline { 3 - 5 } $\begin{array}{l}\text { Amount of } \\
\mathrm{Ac}_{2} \mathrm{O} / \mathrm{ml}\end{array}$ & $\begin{array}{l}\text { Yield of dinitro- } \\
\text { toluenes }(\%)^{b}\end{array}$ & $2,4-$ & $2,6-$ & $2,4-: 2,6-$ ratio $^{d}$ \\
\hline 0 & 99 & 74 & 26 & 2.9 \\
1.0 & 97 & 78 & 22 & 3.5 \\
2.5 & 98 & 84 & 16 & 5.2 \\
3.5 & 99 & 94 & 5.5 & 17 \\
4.0 & 98 & 94 & 5.4 & 17
\end{tabular}

${ }^{a}$ Reactions carried out using $\mathrm{H} \beta(1.0 \mathrm{~g}), o$-nitrotoluene $(17.5 \mathrm{mmol})$, nitric acid $(17.5 \mathrm{mmol}$ of $90 \%)$, TFAA $(3.5 \mathrm{ml}, 24 \mathrm{mmol})$ and acetic anhydride (see table) at $-10^{\circ} \mathrm{C}$ for 2 hours. ${ }^{b}$ By quantitative GC. ${ }^{c}$ Proportions of isomers (\%) in the product mixture, determined by GC (to 2 significant figures). ${ }^{d}$ Ratio calculated directly from GC data.

Table 2 Effect of amount of $\mathrm{H} \beta^{a}$

\begin{tabular}{lllll}
\hline \multirow{2}{*}{$\begin{array}{l}\text { Amount } \\
\text { of } \mathrm{H} \beta / \mathrm{g}\end{array}$} & $\begin{array}{l}\text { Yield of dinitro- } \\
\text { toluenes }(\%)^{b}\end{array}$ & $2,4-$ & $2,6-$ & $2,4: 2,6$-ratio ${ }^{d}$ \\
\cline { 3 - 5 } 0 & 16 & 66 & 34 & 2.0 \\
0.25 & 93 & 84 & 16 & 5.1 \\
0.5 & 94 & 88 & 12 & 7.3 \\
0.75 & 93 & 93 & 6.5 & 14 \\
1.0 & 99 & 94 & 5.5 & 17 \\
1.5 & 99 & 94 & 5.4 & 17 \\
2.0 & 99 & 94 & 5.5 & 17 \\
3.0 & 99 & 94 & 5.4 & 17
\end{tabular}

${ }^{a}$ Reactions were carried out using $\mathrm{H} \beta$ (see table), $o$-nitrotoluene (17.5 $\mathrm{mmol})$, nitric acid $(17.5 \mathrm{mmol}$ of $90 \%)$, TFAA $(3.5 \mathrm{ml}, 24 \mathrm{mmol})$ and acetic anhydride $(3.5 \mathrm{ml}, 37 \mathrm{mmol})$ at $-10{ }^{\circ} \mathrm{C}$ for 2 hours. ${ }^{b}$ By quantitative GC. ${ }^{c}$ Proportions of isomers (\%) in the product mixture, determined by GC (to 2 significant figures). ${ }^{d}$ Ratio calculated directly from GC data.

As indicated above, the trifluoroacetyl nitrate mixture in the presence of zeolite quantitatively nitrated $o$-nitrotoluene with a slight improvement in the regioselectivity towards the 2,4isomer (Table 1). On addition of acetic anhydride to the mixture [eqn. (2)], prior to addition of the substrate, the selectivity<smiles>Cc1ccccc1[N+](=O)[O-]</smiles>

was improved further without detrimental effect on the overall reactivity. No further improvement in selectivity was observed after the volume of acetic anhydride added was about equal to that of the minimum amount of trifluoroacetic anhydride (Table 1).

The effect of the amount of zeolite was next investigated for a $17.5 \mathrm{mmol}$ scale reaction using the optimal amount of acetic anhydride discovered above. As was expected, the amount of zeolite present directly affects reactivity and selectivity, with the highest ratio of 2,4-:2,6- being around 17:1 (Table 2). The selectivity steadily improved with increasing amount of zeolite until $c a$. $1 \mathrm{~g}$ was used, with larger amounts having only marginal additional effects.

In order to check whether the standard period of 2 hours used for the reactions thus far was actually required, a reaction using the same conditions as those described in Table 2, with $1 \mathrm{~g}$ of zeolite, was stopped after 1 hour. The yield of dinitrotoluenes was only $90 \%$, and the proportion of 2,4-:2,6- was almost unchanged (16.6). Therefore, it appeared sensible to continue to use 2 hours for the reaction period. 
Table 3 Effect of reaction temperature ${ }^{a}$

\begin{tabular}{rllll}
\hline & & \multicolumn{3}{l}{ Proportions of isomers ${ }^{c}$} \\
\cline { 3 - 5 } Temp. ${ }^{\circ} \mathrm{C}$ & $\begin{array}{l}\text { Yield of dinitro- } \\
\text { toluenes }(\%)^{b}\end{array}$ & $2,4-$ & $2,6-$ & $2,4-: 2,6-$ ratio $^{d}$ \\
\hline-78 & 2 & 92 & 8.4 & 11 \\
-41 & 71 & 93 & 6.7 & 13 \\
-10 & 98 & 94 & 5.5 & 17 \\
0 & 95 & 94 & 5.6 & 16 \\
20 & 90 & 94 & 5.5 & 17
\end{tabular}

${ }^{a}$ Reactions were carried out using $\mathrm{H} \beta$ (1.0 g), o-nitrotoluene (17.5 mmol), nitric acid (17.5 mmol of 90\%), TFAA (3.5 ml, $24 \mathrm{mmol})$ and acetic anhydride $(3.5 \mathrm{ml}, 37 \mathrm{mmol})$ at the temperature stated in the table for 2 hours. ${ }^{b}$ By quantitative GC. ${ }^{c}$ Proportions of isomers (\%) in the product mixture, determined by GC (to 2 significant figures). ${ }^{d}$ Ratio calculated directly from $\mathrm{GC}$ data.

The effect of temperature on the reaction was also investigated and the results are reported in Table 3 . At $20^{\circ} \mathrm{C}$, a decrease in yield was observed but with no effect on selectivity. This can be explained by loss from the system of trifluoroacetic anhydride, which has a boiling point of $39.5^{\circ} \mathrm{C}$. Therefore, lower temperatures are preferable. However, if the temperature is too low, then a marked decrease in reactivity is observed and so the optimal temperature was found to be around $-10{ }^{\circ} \mathrm{C}$ (cooling in an ice-salt bath).

$p$-Nitrotoluene could also be nitrated with the optimised system used for $o$-nitrotoluene, but this reaction is much slower and so for quantitative yields it is quicker to use trifluoroacetic anhydride, nitric acid and zeolite $\mathrm{H} \beta$ (i.e. without addition of acetic anhydride). It was of interest to know if a common system could be developed, which could then be applied to the direct dinitration of toluene without the need to isolate any intermediate. Therefore, attention was next paid to this problem.

\section{Dinitration of toluene in a single step}

The dinitration of toluene using mixed acids gives a mixture of isomers from the further nitration of the $o-, m$ - and $p$-nitrotoluenes formed as intermediates. The predominant products are 2,4- and 2,6-dinitrotoluenes, formed in a ratio of $4: 1$. This ratio has been improved to $9: 1$ using claycop, nitric acid and acetic anhydride, ${ }^{5}$ and to $14: 1$ using nitric acid and zeolite $\mathrm{H} \beta,{ }^{8}$ but yields are also lower in such systems. We attempted to carry out a single step nitration of toluene [eqn. (3)] under

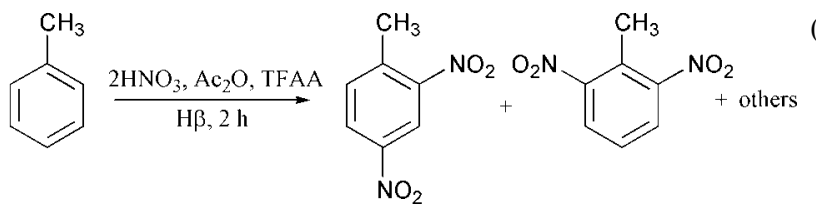

conditions similar to those developed for the selective nitration of $o$-nitrotoluene, but using two equivalents of nitric acid. We also used a slightly smaller quantity of acetic anhydride, so as not to inhibit too much the further nitration of the less reactive $p$-nitrotoluene. The results are shown in Table 4.

As the results in Table 4 show, the reaction was very selective, producing dinitrotoluenes in almost quantitative yield and with high selectivity using $1 \mathrm{~g}$ zeolite, and still providing good selectivity even when only $0.5 \mathrm{~g}$ of zeolite was used. Nevertheless, the ratio of 2,4-:2,6-isomers was less than would be predicted if the first step were to give $o$ - and $p$-nitrotoluenes in the published ratio for nitric acid-acetic anhydride- $\mathrm{H} \beta$ (ca. 4:1), and the $p$-nitrotoluene were to give only 2,4-dinitrotoluene while the $o$-nitrotoluene were to react as described in Tables $1-3$, giving a $17: 1$ ratio of $2,4-: 2,6$-isomers. In this case the
Table 4 Dinitration of toluene according to eqn. (3) ${ }^{a}$

\begin{tabular}{lllll}
\hline & & \multicolumn{3}{c}{ Yields of isomers $^{c}$} \\
\cline { 3 - 5 } $\begin{array}{l}\text { Amount } \\
\mathrm{H} \beta / \mathrm{g}\end{array}$ & $\begin{array}{c}\text { Yield of dinitro- } \\
\text { toluenes }(\%)^{b}\end{array}$ & $2,4-$ & $2,6-$ & $2,4-: 2,6-$ ratio $^{d}$ \\
\hline 0.5 & 98 & 89 & 6.4 & 14 \\
1.0 & 98 & 92 & 3.8 & 24
\end{tabular}

${ }^{a}$ Reactions were carried out using $\mathrm{H} \beta$ (see table), nitric acid $(35 \mathrm{mmol}$ of $90 \%$ ), acetic anhydride $(24 \mathrm{mmol})$, trifluoroacetic anhydride (24 $\mathrm{mmol})$ and toluene $(17.5 \mathrm{mmol})$ at $-10{ }^{\circ} \mathrm{C}$ for 2 hours. ${ }^{b}$ By quantitative GC. ${ }^{c}$ Absolute yields of isomers $(\%)$ in the product mixture, determined by GC (to 2 significant figures); other isomers derived primarily from $m$-nitrotoluene make up the rest. ${ }^{d}$ Ratio calculated directly from GC data.

Table 5 Effect of addition of extra zeolite during the second step ${ }^{a}$

\begin{tabular}{lllll}
\hline & & \multicolumn{3}{l}{ Proportions of isomers ${ }^{c}$} \\
\cline { 3 - 5 } Zeolite & $\begin{array}{l}\text { Yield of dinitro- } \\
\text { toluenes }(\%)^{b}\end{array}$ & $2,4-$ & $2,6-$ & $2,4-: 2,6-$ ratio $^{d}$ \\
\hline HY & 98 & 95 & 2.5 & 38 \\
H $\beta$ & 99 & 96 & 1.4 & 70
\end{tabular}

${ }^{a}$ Reactions were carried out using $\mathrm{H} \beta(1.0 \mathrm{~g})$, nitric acid $(17.5 \mathrm{mmol}$ of $90 \%$, acetic anhydride $(24 \mathrm{mmol})$ and toluene $(17.5 \mathrm{mmol})$ at room temperature for 30 minutes for the first step; then extra nitric acid $(17.5 \mathrm{mmol}, 90 \%)$, TFAA $(24 \mathrm{mmol})$ and extra zeolite $(1.0 \mathrm{~g})$ at $-10{ }^{\circ} \mathrm{C}$ for the second step. ${ }^{b}$ By quantitative GC. ${ }^{c}$ Proportions of isomers by GC (to 2 significant figures), other isomers derived predominantly from $m$-nitrotoluene make up the rest. ${ }^{d}$ Ratio calculated directly from GC data.

ratio expected for the overall process would be in the region of $98: 2$ to $99: 1$. It seemed likely that the problem was a less selective first step in the presence of trifluoroacetic anhydride. Therefore, a two-step sequence, in which trifluoroacetic anhydride was introduced only during the second step, was investigated.

\section{Dinitration of toluene in a two-step process}

It was hoped that use of the highly regioselective system previously developed for the mononitration of toluene ${ }^{4}$ with subsequent addition of the more reactive trifluoroacetic anhydride (TFAA) along with the extra nitric acid for the second step [eqn. (4)], would further improve the regioselectivity.

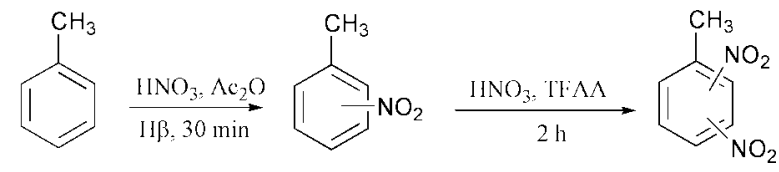

Indeed, when the reaction was carried out in this way, a $98 \%$ yield of dinitrotoluenes, with a $38: 1$ ratio of 2,4-:2,6-isomers was obtained.

To try to improve this selectivity even further we investigated the effect of addition of extra zeolite for the second step, and these results are shown in Table 5. Zeolite HY and zeolite $\mathrm{H} \beta$, which are both large pore zeolites, were tried, but $\mathrm{H} \beta$ still proved to be the optimal zeolite, giving the highest selectivity towards the 2,4-isomer.

Using a two-step reaction, we have now improved the regioselectivity of the dinitration of toluene to give a ratio of 2,4-:2,6- of $70: 1$, with quantitative yields after 2 hours at around $-10{ }^{\circ} \mathrm{C}$.

Pure 2,4-dinitrotoluene could be isolated in $90 \%$ yield from this system simply by filtering the zeolite, concentrating the mother liquor, and recrystallization from acetone. 
Table 6 Nitration of $\mathrm{PhX}$ according to eqn. (5) ${ }^{a}$

\begin{tabular}{llccc}
\hline $\mathrm{X}$ & Yield $(\%)^{b}$ & $\mathbf{2}^{c}$ & $\mathbf{3}^{c}$ & $\mathbf{4}^{c}$ \\
\hline $\mathrm{NO}_{2}$ & 72 & 5 & 88 & 7 \\
$\mathrm{COOH}$ & $86^{d}$ & $2^{d}$ & $97^{d}$ & $1^{d}$ \\
$\mathrm{CN}$ & 54 & 13 & 74 & 13 \\
\hline $\mathrm{F}$ & $>99$ & 8 & 0 & 92 \\
$\mathrm{Cl}$ & $>99$ & 15 & $<1$ & 84 \\
$\mathrm{Br}$ & $>99$ & 15 & 0 & 85
\end{tabular}

${ }^{a}$ Reactions carried out using $\mathrm{H} \beta$ (1 g), substrate $(17.5 \mathrm{mmol})$, nitric acid $(17.5 \mathrm{mmol}$ of $90 \%)$ and TFAA $(3.5 \mathrm{ml}, 17.5 \mathrm{mmol})$ at $-10{ }^{\circ} \mathrm{C}$ for 2 hours. ${ }^{b}$ Total yield of mononitrated products by quantitative GC. ${ }^{c}$ Proportions of isomers $(\%)$ in the product mixture, determined by GC. ${ }^{d}$ By quantitative HPLC.

\section{Nitration of other deactivated aromatic compounds}

In view of the success in achieving selective conversion of $o$ nitrotoluene into 2,4-dinitrotoluene, and of the direct conversion of toluene into 2,4-dinitrotoluene, it was of interest to investigate the nitration of other deactivated substrates using the reagent systems developed. Three reagent systems were considered: (i) nitric acid-acetic anhydride- $\mathrm{H} \beta$, as used in our mononitration of toluene and other moderately active substrates; ${ }^{4}$ (ii) nitric acid-trifluoroacetic anhydride- $\mathrm{H} \beta$, as used in the further nitration of $p$-nitrotoluene; and (iii) nitric acid-trifluoroacetic anhydride-acetic anhydride- $\mathrm{H} \beta$, as used in the selective nitration of $o$-nitrotoluene.

The nitric acid-acetic anhydride- $\mathrm{H} \beta$ system has already been shown to be capable of nitrating modestly deactivated substrates such as halogenobenzenes, albeit more slowly and in somewhat lower yields than for toluene. ${ }^{4}$ However, when it was used under the standard conditions for the nitration of $o$-nitrotoluene, a substrate bearing both an activating and a deactivating substituent, it gave only $20 \%$ conversion, and with slightly lower selectivity for the 2,4-dinitrotoluene isomer than when trifluoroacetic anhydride was used. Furthermore, under the same conditions it gave only a small amount of dinitrobenzenes when applied to nitrobenzene, a substrate bearing only a single, strongly deactivating substituent. In view of these results, no further attention was paid to this reagent system.

A series of deactivated monosubstituted benzenes was subjected to the system nitric acid-trifluoroacetic anhydride$\mathrm{H} \beta$ [eqn. (5)]. These substrates included several meta-directing<smiles>[X]c1ccccc1</smiles>

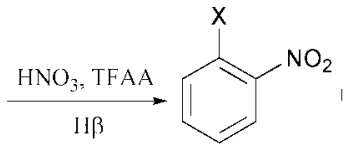<smiles>[X]c1cccc([N+](=O)[O-])c1</smiles><smiles>[X]c1ccc([N+](=O)[O-])cc1</smiles>

deactivating groups, as well as several ortho-directing halogenobenzenes. The results are shown in Table 6.

As the results in Table 6 show, the halogenobenzenes were all quantitatively nitrated under the reaction conditions. The selectivities for the para-isomer were good, but somewhat less than for the previously reported reaction involving acetic anhydride, ${ }^{4}$ which is not surprising given the greater activity of the present reagent system. Reasonable yields of mononitrated products were also obtained from the meta-directing substrates under the standard conditions, and individual optimization of the reaction conditions would probably allow quantitative conversion in such cases. There was some increase in the amount of both the ortho- and para-isomers, in comparison to mixed acid nitration, for two of the substrates, but meta-nitro products were still vastly predominant.
Table 7 Nitration of $\mathrm{PhX}$ according to eqn. (6) ${ }^{a}$

\begin{tabular}{llccc}
\hline $\mathrm{X}$ & ${\text { Yield }(\%)^{b}}^{b}$ & $\mathbf{2}^{c}$ & $\mathbf{3}^{c}$ & $\mathbf{4}^{c}$ \\
\hline $\mathrm{NO}_{2}$ & 25 & 2 & 91 & 7 \\
$\mathrm{COOH}$ & $74^{d}$ & $11^{d}$ & $79^{d}$ & $10^{d}$ \\
$\mathrm{CN}$ & 17 & 10 & 71 & 19 \\
\hline $\mathrm{F}$ & $>99$ & $<1$ & 0 & 99 \\
$\mathrm{Cl}$ & $>99$ & 7 & $<1$ & 92 \\
$\mathrm{Br}$ & 97 & 11 & 0 & 89
\end{tabular}

${ }^{a}$ Reactions carried out using $\mathrm{H} \beta$ (1.0 g), substrate $(17.5 \mathrm{mmol})$, nitric acid $(17.5 \mathrm{mmol}$ of $90 \%)$, TFAA $(3.5 \mathrm{ml}, 17.5 \mathrm{mmol})$ and acetic anhydride $(3.5 \mathrm{ml})$ at $-10{ }^{\circ} \mathrm{C}$ for 2 hours. ${ }^{b}$ By quantitative GC. ${ }^{c}$ Proportions of isomers $(\%)$ in the product mixture, determined by GC. ${ }^{d}$ By quantitative HPLC.

Finally, attention was turned to the system nitric acid-trifluoroacetic anhydride-acetic anhydride-H $\beta$ [eqn. (6)]. The

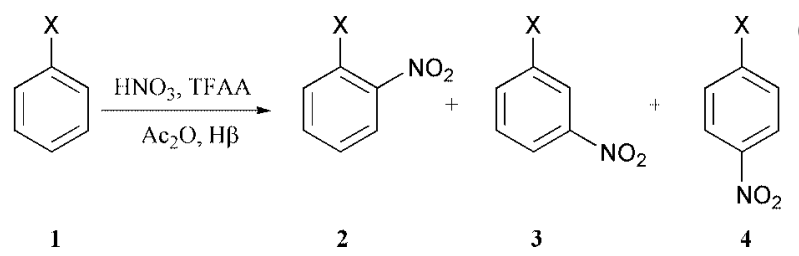

same substrates were used, and the results of subjecting all six substrates to a standard set of conditions are given in Table 7.

With the halogenobenzenes, the reactivity was largely unaffected by the addition of acetic anhydride, reactions being essentially quantitative under the standard conditions. However, improvement in para selectivity was observed compared with the reaction using only trifluoroacetic anhydride. From the mixed-acid nitration of chlorobenzene, the $p: o$ ratio is 2 , which is also observed in nitration using nitrogen dioxide, oxygen and $\mathrm{Fe}(\mathrm{acac})_{3} \cdot{ }^{11}$ This ratio has been improved using acetyl nitrate and zeolite $\mathrm{H} \beta$ to $p: o=13,{ }^{4}$ and now the nitric acid-trifluoroacetic anhydride-acetic anhydride- $\mathrm{H} \beta$ system has been found to give the same high regioselectivity (Table 7) but in higher yield, more quickly and at a reduced temperature.

In the case of the meta-directing substrates, the reaction rates were slower in the presence of acetic anhydride and the yields were lower under the standard conditions. There was, in most cases, an increase in the proportion of the para-isomer, to a remarkable $19 \%$ in the case of benzonitrile, presumably as a result of shape-selectivity imposed on the transition state. In the case of benzoic acid, there was also an increase in the proportion of the ortho-isomer, possibly resulting from rearrangement of benzoyl nitrate formed in situ. However, in all cases the meta-isomer was still vastly predominant, so there seems little reason to carry out reactions of deactivated, monosubstituted meta-directing substrates with this reagent system.

The remarkably high proportion of para-product from benzonitrile, and the excellent selectivity for 2,4-dinitrotoluene on nitration of 2-nitrotoluene with this system, however, encouraged us to try the reaction with 2-methylbenzonitrile. The reaction proved to be successful [eqn. (7)], giving almost<smiles>CC1=C(C#N)C=CCC1</smiles>

quantitative conversion into mononitro products and a 2,4-:2,6-cyanonitrotoluene ratio of 6.5, compared with only 1.8 in the case of mixed acids. 


\section{Conclusions}

The use of nitrating systems containing nitric acid, trifluoroacetic anhydride and zeolite $\mathrm{H} \beta$ allows nitration of deactivated aromatic substrates. For monosubstituted benzenes carrying a meta-directing group $\left(\mathrm{NO}_{2}, \mathrm{CO}_{2} \mathrm{H}, \mathrm{CN}\right)$, this system provides high yields of the mononitrated derivatives. The normal preference for meta-substituted product is seen, though in some cases there may be a small increase in the amount of orthoand/or para-products compared with mixed acid systems. The nitric acid-trifluoroacetic anhydride- $\mathrm{H} \beta$ system is also useful for nitration of 4-nitrotoluene to 2,4-dinitrotoluene.

For deactivated substrates of somewhat higher reactivity, such as halogenobenzenes, 2-nitrotoluene and 2-methylbenzonitrile, it is better to use a system that has been moderated by addition of acetic anhydride, in a volume approximately equal to that of the trifluoroacetic anhydride. The lower activity of the system allows greater control over the selectivity. Halogenobenzenes give almost quantitative mononitration with very high selectivity for the para-nitro products. 2-Nitrotoluene gives very high selectivity for 2,4-dinitrotoluene, while 2-methylbenzonitrile gives around $87 \%$ of 2-cyano-4-nitrotoluene. In both the latter two cases the selectivity for nitration at the 4-position (relative to the methyl) is much greater than for mixed acid systems.

By adaptation of the latter process it has proved possible to double nitrate toluene selectively without isolation of any intermediate. If the reaction is carried out in a single step, the yield of 2,4-dinitrotoluene is $92 \%$ and the ratio of $2,4-: 2,6$ dinitrotoluenes is $25: 1$. The yield can be improved to $96 \%$ and the selectivity to $70: 1$ by carrying out the nitration in two stages, with the trifluoroacetic anhydride added only for the second stage. However, the reaction is still conducted all in one flask and without isolation of any intermediate. This is easily the most selective double nitration of toluene ever recorded

It is recognised that trifluoroacetic anhydride's volatility, toxicity and cost may render it unattractive for larger scale commercial processes, but in our previous work with acetic anhydride alone we showed that acetic acid could be recovered quantitatively, ${ }^{4}$ and recovery should be equally easy with the more volatile trifluoroacetic acid. In the earlier work we also showed that the zeolite could be reused several times, which improves the economics. The methods demonstrated here could therefore prove attractive, particularly for smaller scale syntheses.

\section{Experimental}

Nitration procedure: nitrations using nitric acid, trifluoroacetic anhydride, acetic anhydride and zeolite $\mathrm{H} \beta$

A cooled mixture (ice-salt bath, bath temp. $=-10{ }^{\circ} \mathrm{C}$ ) of acetic anhydride $(3.5 \mathrm{ml}, 37 \mathrm{mmol})$ and trifluoroacetic anhydride $(3.5 \mathrm{ml}, 17.5 \mathrm{mmol})$ was added to a stirring mixture of zeolite $\mathrm{H} \beta(1.0 \mathrm{~g}, \mathrm{Si}: \mathrm{Al}$ ratio $=12.5)$ and nitric acid $(1.23 \mathrm{~g}, 90 \%$, $17.5 \mathrm{mmol}$ ) and stirred for $5 \mathrm{~min}$ at constant temperature. The substrate $(17.5 \mathrm{mmol})$ was then added and the mixture was stirred for $2 \mathrm{~h}$ at around $-10{ }^{\circ} \mathrm{C}$. The zeolite was removed by suction filtration and washed with copious amounts of acetone. The mother liquors were combined.

The yields and compositions of the product mixtures were analysed by quantitative GC or HPLC using an internal standard (hexadecane or heptadecane in the case of GC and 4-hydroxybenzaldehyde in the case of HPLC), and identities were proved by comparison with authentic samples. The results are given in Table 7.

\section{Nitration procedure: nitrations using nitric acid, trifluoroacetic} anhydride and zeolite $\mathbf{H \beta}$

Trifluoroacetic anhydride $(3.5 \mathrm{ml}, 17.5 \mathrm{mmol})$ was added to a stirring mixture of $\mathrm{H} \beta(1.0 \mathrm{~g}, \mathrm{Si}: \mathrm{Al}=12.5)$ and nitric acid
$(1.23 \mathrm{~g}, 90 \%, 17.5 \mathrm{mmol})$ with cooling in an ice-salt bath (temp. $=-10{ }^{\circ} \mathrm{C}$ ) and the mixture was stirred for $5 \mathrm{~min}$ at constant temperature. The substrate $(17.5 \mathrm{mmol})$ was then added and the mixture was stirred for $2 \mathrm{~h}$ at around $-10{ }^{\circ} \mathrm{C}$. The zeolite was removed by suction filtration and washed with copious amounts of acetone. The mother liquors were combined.

The yields and compositions of the product mixtures were analysed by quantitative GC or HPLC using an internal standard (hexadecane or heptadecane in the case of GC and 4-hydroxybenzaldehyde in the case of HPLC), and identities were proved by comparison with authentic samples. The results are given in Table 6 .

\section{Nitration procedure: one-step nitration of toluene}

A cooled mixture (ice-salt bath) of acetic anhydride (2.48 g, $24 \mathrm{mmol})$ and trifluoroacetic anhydride $(3.5 \mathrm{ml}, 24 \mathrm{mmol})$ was added to a stirring mixture of zeolite $\mathrm{H} \beta(1.0 \mathrm{~g}, \mathrm{Si}: \mathrm{Al}=12.5)$ and nitric acid $(2.46 \mathrm{~g}, 90 \%, 35 \mathrm{mmol})$ and stirred for $5 \mathrm{~min}$ at constant temperature. Toluene $(1.61 \mathrm{~g}, 17.5 \mathrm{mmol})$ was then added and the mixture was stirred for $2 \mathrm{~h}$ at around $-10^{\circ} \mathrm{C}$. The zeolite was removed by suction filtration and washed with copious amounts of acetone. The mother liquors were combined.

The yields and composition of product mixtures were analysed by quantitative GC using hexadecane as the internal standard. The results are shown in Table 4.

\section{Nitration procedure: two-step nitration of toluene}

Acetic anhydride $(2.48 \mathrm{~g}, 24 \mathrm{mmol})$ was added to a stirring mixture of nitric acid $(1.23 \mathrm{~g}, 90 \%, 17.5 \mathrm{mmol})$ and $\mathrm{H} \beta(0.5 \mathrm{~g}$, $\mathrm{Si}: \mathrm{Al}=12.5)$ at $0{ }^{\circ} \mathrm{C}$ and the mixture was stirred for $5 \mathrm{~min}$ at constant temperature. Toluene $(1.61 \mathrm{~g}, 17.5 \mathrm{mmol})$ was then added dropwise and the mixture was allowed to warm to room temperature. After $30 \mathrm{~min}$, the mixture was cooled to around $-10^{\circ} \mathrm{C}$ (ice-salt bath), fresh $\mathrm{H} \beta(1 \mathrm{~g}, \mathrm{Si}: \mathrm{Al}=12.5)$, nitric acid $(1.23 \mathrm{~g}, 90 \%, 17.5 \mathrm{mmol})$ and trifluoroacetic anhydride $(3.5 \mathrm{ml}$, $24 \mathrm{mmol}$ ) were added, and the mixture was stirred for $2 \mathrm{~h}$ at around $-10{ }^{\circ} \mathrm{C}$. The zeolite was removed by suction filtration and washed with copious amounts of acetone. The mother liquors were combined.

The yields and composition of product mixtures were analysed by quantitative GC using hexadecane as the internal standard. The yield of 2,4-dinitrotoluene was $96 \%$ and the ratio of 2,4- : 2,6-dinitrotoluenes was 70:1.

Pure 2,4-dinitrotoluene ( $>99 \%$ purity by GC) was isolated from the reaction mixture in $90 \%$ yield by simply concentrating the mother liquor and recrystallization from analytical grade acetone.

\section{GC analysis}

All GC analyses were carried out on a HP5890 Series II chromatograph with a HP 3395 integrator, using a Carbowax column, $30 \mathrm{~m} \times 0.32 \mathrm{~nm} \times 0.25 \mu \mathrm{m}$.

\section{HPLC analysis}

HPLC analysis was carried out using a Luna $3 \mu \mathrm{m}$ phenyl hexyl column (supplied by Phenomenex), using water-acetonitrile

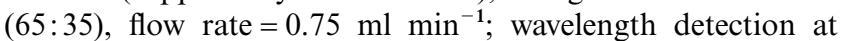
$254 \mathrm{~nm}$.

\section{Acknowledgements}

This work was supported by the U.K. Defence Evaluation and Research Agency, whose financial assistance is gratefully acknowledged. 


\section{References}

1 (a) G. A. Olah, Nitration: Methods and Mechanisms, VCH, New York, 1989; (b) K. Schofield, Aromatic Nitration, Cambridge University Press, Cambridge, 1980; (c) R. Taylor, Electrophilic Aromatic Substitution, John Wiley and Sons, Chichester, 1990.

2 Chemistry of Waste Minimisation, ed. J. H. Clark, Chapman and Hall, London, 1995.

3 P. Laszlo, Acc. Chem. Res., 1986, 19, 121; P. Laszlo and J. Vandormael, Chem. Lett., 1988, 1843; P. Laszlo, A. Cornelis and A. Gerstmans, Chem. Lett., 1988, 1839; A. Cornelis, L. Delaude, A. Gerstmans and P. Laszlo, Tetrahedron Lett., 1988, 29, 5657.

4 K. Smith, A. Musson and G. A. DeBoos, J. Org. Chem., 1998, 63, 8448.

5 B. Carvalheiro, P. Laszlo, A. Cornelis and M. Marcelo-Curto,
PCT Int. Appl. WO 94/19310 1994; B. Gigante, A. Prazeres, M. Marcelo-Curto, A. Cornelis and P. Laszlo, J. Org. Chem., 1995, 60, 3445 .

6 F. J. Waller, A. G. Barrett, D. C. Braddock and D. Ramprasad, Tetrahedron Lett., 1998, 39, 1641.

7 H. Suzuki, T. Murashima, K. Shimizu and K. Tsukamoto, J. Chem. Soc., Chem. Commun., 1991, 1049.

8 D. Vassena, A. Kogelbauer, R. Prins and J. Armor, Abstracts from EuropaCat-IV, Rimini, 1999, p. 222.

9 K. Smith, Bull. Soc. Chim. Fr., 1989, 272; K. Smith, K. Fry, M. Butters and B. Nay, Tetrahedron Lett., 1989, 30, 5333.

10 E. J. Bourne, M. Stacey, J. C. Tatlow and J. M. Tedder, J. Chem. Soc., 1952, 1695.

11 H. Suzuki, S. Yonezawa, N. Nonoyama and T. Moi, J. Chem. Soc., Perkin Trans. 1, 1996, 2385. 